\title{
Recent Developments in Computed Tomography for Urolithiasis: Diagnosis and Characterization
}

\author{
P. D. Mc Laughlin, L. Crush, M. M. Maher, and O. J. O’Connor \\ Department of Radiology, Cork University Hospital and University College Cork, Cork, Ireland \\ Correspondence should be addressed to L. Crush, lcrush03@gmail.com
}

Received 30 April 2012; Accepted 20 July 2012

Academic Editor: Ahmed M. Shoma

Copyright ( $) 2012$ P. D. Mc Laughlin et al. This is an open access article distributed under the Creative Commons Attribution License, which permits unrestricted use, distribution, and reproduction in any medium, provided the original work is properly cited.

Objective. To critically evaluate the current literature in an effort to establish the current role of radiologic imaging, advances in computed tomography (CT) and standard film radiography in the diagnosis, and characterization of urinary tract calculi. Conclusion. CT has a valuable role when utilized prudently during surveillance of patients following endourological therapy. In this paper, we outline the basic principles relating to the effects of exposure to ionizing radiation as a result of CT scanning. We discuss the current developments in low-dose CT technology, which have resulted in significant reductions in CT radiation doses (to approximately one-third of what they were a decade ago) while preserving image quality. Finally, we will discuss an important recent development now commercially available on the latest generation of CT scanners, namely, dual energy imaging, which is showing promise in urinary tract imaging as a means of characterizing the composition of urinary tract calculi.

\section{Introduction}

Since its first description by Smith et al. in 1995, noncontrast computed tomography (CT) of the urinary tract has become the imaging investigation of choice in patients with acute renal colic [1-3] due to its significantly higher sensitivity and specificity for detection of urinary stones when compared with plain radiography and intravenous urography and also due to superior capability for accurate characterization of the size and location of obstructing urinary calculi, thus allowing clinicians to predict the likelihood of spontaneous passage $[4,5]$.

Although CT is extremely valuable in imaging residual calculi and small fragments following endourological treatment, there are accepted limitations inherent to the technique, which are important in this setting.

(1) Limited spatial resolution: CT has limited spatial resolution; therefore, its negative predictive value in fully excluding submillimeter calculi and small stone fragments is significantly less than its negative predictive value in excluding larger calculi ( $>4 \mathrm{~mm})$.
(2) Use of ionizing radiation: patients may present with their first episode of renal colic at a young age, and recurrence in subsequent years is reported in 25$50 \%$ of cases [6-8]. Repeated use of CT in these patients can result in a substantial cumulative dose; Ferrandino et al. reported a large cumulative effective radiation dose attributable to $\mathrm{CT}$ scanning during short-term followup of patients with urinary calculi [9]. Therefore, judicious and informed use of this modality is mandatory when imaging residual stones following endourological therapy in an effort to minimize the potential for significant exposures to ionizing radiation.

Despite these challenges, CT has a valuable role when utilized prudently during surveillance of patients following endourological therapy. In this paper, we outline the basic principles relating to the effects of exposure to ionizing radiation as a result of $\mathrm{CT}$ scanning. We discuss the current developments in low-dose CT technology, which have resulted in significant reductions in CT radiation doses (to approximately one-third of what they were a decade ago) while preserving image quality. Finally, we will discuss an 
important recent development now commercially available on the latest generation of CT scanners, namely dual energy imaging, which is showing promise in urinary tract imaging as a means of characterizing the composition of urinary tract calculi.

\section{Ionizing Radiation}

One of the most feared adverse events associated with exposure to ionizing radiation is carcinogenesis, which is a stochastic effect, that is to say, it is random. Cancer induction does not exhibit an upper or lower threshold of occurrence, and the probability of cancer induction is variable [10]. The overall risk of cancer is currently believed to be small, but a cause for concern with regard to radiation exposures in the diagnostic imaging range is that there is no radiation dose below which cancer induction does not occur. In addition, it is important to highlight that carcinogenesis may transpire many years following exposure, and it is accepted that exposure to ionizing radiation in early life magnifies the risk of tumor induction [11].

The general population is becoming increasingly aware and concerned about the potential health risks associated with ionizing radiation [12]. This is due to recent widespread press and media coverage highlighting the potential increase in cancer risk from exposure to ionizing radiation related to the increasing use of CT [13]. Today's young and educated patients with renal colic may in particular be conscious of exposure to ionizing radiation as a result of diagnostic imaging, and cancer induction is their primary concern. Their apprehension is not unfounded as large cumulative effective doses of $>50 \mathrm{mSv}$ during short-term followup $(<1$ year) were reported by Ferrandino et al. in $20 \%$ of patients with renal colic [9], and previous studies have suggested that cumulative exposure to similar levels of ionizing radiation (75 mSv) from sources other than CT may increase mortality due to cancer by $7.3 \%$ [11].

\section{Low-Dose CT: An Update}

Many studies have examined the diagnostic efficacy of lowdose CT in the setting of renal colic, and a variety of protocols have described that result in effective radiation dose reductions of up to $95 \%$ from greater than $10 \mathrm{mSv}$ to as low as $0.5-3.5 \mathrm{mSv}$ [14-24]. Low-dose CT is uniformly associated with an increase in image noise, but successes in dose reduction in the setting of renal colic have been aided by the inherent high contrast of renal calculi against the relatively low-density soft tissues surrounding the urinary tract.

In the past, fixed CT settings (e.g., $\mathrm{kVp}$ and $\mathrm{mAs}$ ) resulted in lower attenuating areas such as the midabdomen receiving the same radiation exposure as higher attenuating regions such as the pelvis. This was an inefficient method of image acquisition and of imparting radiation exposure to patients as some regions were overirradiated, without any benefit in terms of image quality, while other regions were potentially underexposed, increasing image noise and reducing image quality. Automatic tube current modulation (ATCM) was a major development in CT technology in the last decade. One of the first major papers which evaluated ATCM as a means of optimizing radiation dose at CT reported dose reductions of $32 \%$ in $87 \%$ of CT examinations using ATCM [25]. Reductions in CT dose inherently create an increase in image noise, and the current focus of research and development in the area of CT radiation dose optimization is on the development of noise reduction algorithms to help preserve image quality in CT images acquired at a significantly reduced radiation dose; iterative reconstruction algorithms currently represent the most exciting dose optimizing developments in CT [26] (see Figure 1). Various modifications of iterative reconstruction are being developed and refined by different CT manufacturers including: adaptive statistical iterative reconstruction (ASIR) (General Electric Healthcare, Milwaukee, Wisconsin), sinogram affirmed iterative reconstruction (SAFIRE) (Siemens Healthcare, Erlangen, Germany), iterative reconstruction in image space (IRIS) (Siemens Healthcare, Erlangen, Germany), adaptive iterative dose reduction (AIDR) (Toshiba Medical Systems, Tustin, California), and iDose (Phillips Healthcare, Best, The Netherlands).

Emerging iterative reconstruction algorithms are typically noise efficient and computationally fast, and studies to date have mostly found images with good low-contrast detail, preserved image quality, and have facilitated dose reductions of between 20 and $60 \%$ in a variety of phantom [27-31] and in vivo adult [32-35] studies [36]. Iterative reconstruction will be particularly useful in low-dose CT of the urinary tract where image noise is typically high.

The next step in optimizing image quality in studies acquired at significantly reduced radiation dose is the ongoing development of advanced generations of iterative reconstruction such as model-based iterative reconstruction (MBIR), which is being developed by GE Healthcare. MBIR is a fully iterative reconstruction algorithm, which incorporates a physical model of the CT system into the reconstruction process to characterize the data acquisition process, including noise, beam hardening, and scatter. However, due to limitations in computing power and reconstruction technology, model-based iterative approaches have not been practical for commercial CT scanners until recently as reconstruction times had been exceedingly long.

\section{Detection of Residual Urinary Calculi and Their Fragments}

Studies which initially compared noncontrast CT of the urinary tract with plain radiography, ultrasound, and intravenous urography found significantly increased detection of urinary calculi with noncontrast CT leading to its immediate adoption as the imaging investigation of choice in the setting of suspected urinary tract calculi [1, 37, 38]. A recognized limitation in studies, which report the diagnostic performance of noncontrast CT of the urinary tract, is the choice of gold standard investigation upon which sensitivity and specificity calculations are based. In many studies, 


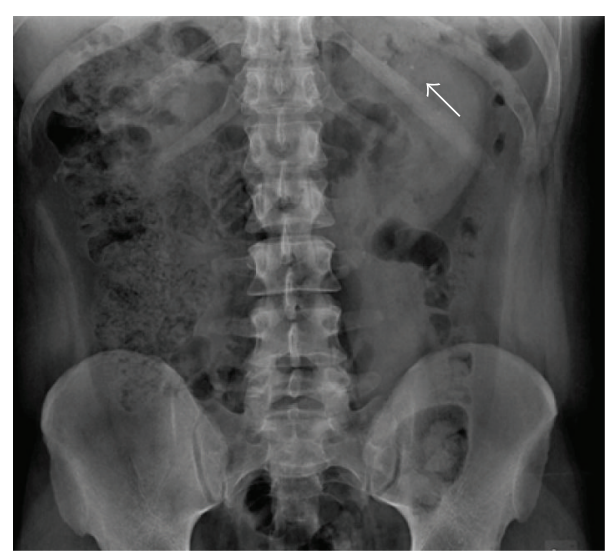

(a)

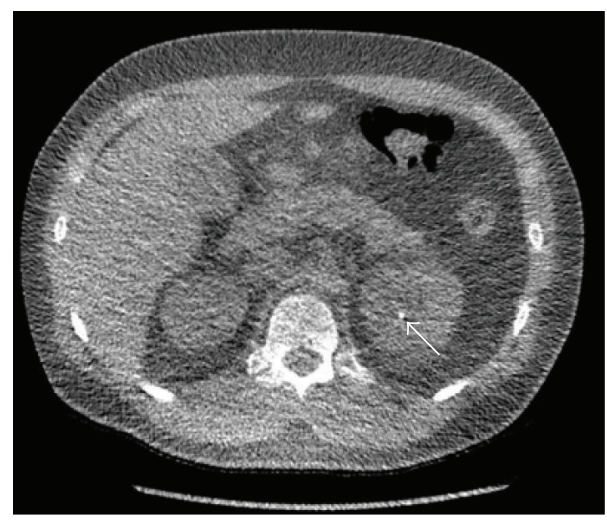

(c)

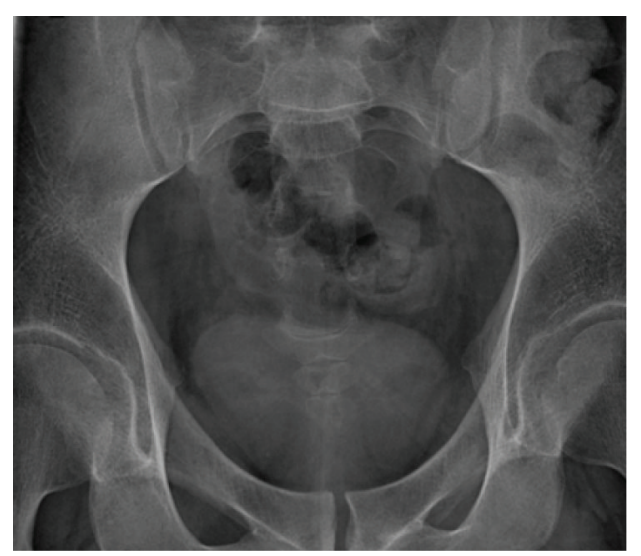

(b)

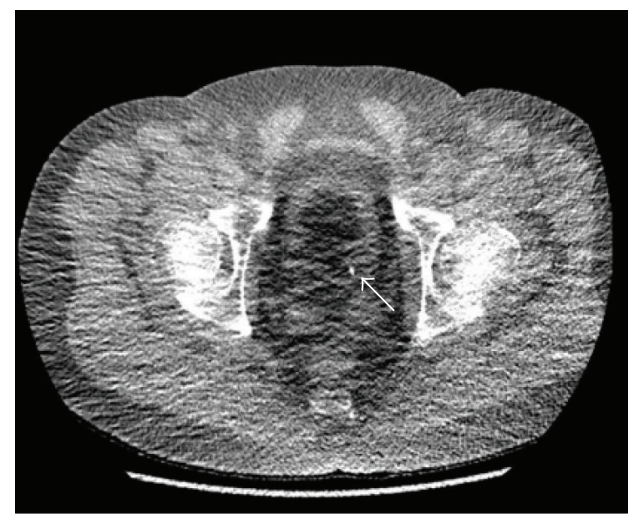

(d)

FIgURE 1: A 25-year-old male presenting with left flank pain. (a) Plain radiograph of the abdomen showing a possible renal calculus in the upper pole of the left kidney (estimated institutional dose $\sim 0.7 \mathrm{mSv}$ ). The coned pelvic radiograph (b) does not demonstrate a calculus in the pelvis. Low-dose CT KUB (effective dose of $0.5 \mathrm{mSv}$ ) (c) and (d) clearly identifies a $5 \mathrm{~mm}$ calculus in the upper pole of the left kidney, as well as a $4 \mathrm{~mm}$ calculus, at the left ureterovesical junction. The latter was not seen on the plain radiograph.

the identification of false negative cases is dependent on additional calculi being subsequently identified with clinical followup, urography, and/or endoscopy in some cases. This may potentially lead to an inappropriately low detection of false negative cases and consequently a spurious increase in the reported sensitivity of noncontrast CT [39].

In relation to the task of completely excluding all residual stones and their small fragments after endourological treatment, CT has recognized limitations. CT has a limited spatial resolution with typical pixel dimensions in the $x-y$ axis measuring $0.7-1 \mathrm{~mm}$ in diameter; a typical slice reconstruction thickness of $2-5 \mathrm{~mm}$ further reduces spatial resolution along the $z$-axis and thereby obscuring small calculi. Reducing the radiation dose imparted during noncontrast CT of the urinary tract also appears to reduce the spatial threshold at which calculi become invisible [39, 40]. Currently, the best available experimental evidence indicating a size threshold for accurate exclusion of calculi is provided by a cadaveric study by Jin et al. who imaged three to five renal stones measuring $2.0-4.0 \mathrm{~mm}$ that were placed in 14 cadaveric human kidneys [39]. The investigators found poor detection of calculi measuring less than $2 \mathrm{~mm}$ on both low-dose (29\% sensitivity) and conventional-dose (47-59\% sensitivity) CT images. Jin et al. conclude that many previous trials examining the diagnostic performance of noncontrast CT of the urinary tract may overestimate the sensitivity and negative predictive value for detection of small urinary calculi measuring less that $2 \mathrm{~mm}$ in size, but calculi measuring greater than $4 \mathrm{~mm}$ were identified in a much higher proportion of cases (95-100\% sensitivity) for both low-dose and conventional-dose CT [39].

Other authors have found reductions in sensitivity and specificity for detecting small calculi $(<3 \mathrm{~mm})$ when low-dose CT techniques were employed [14-24, 40, 41]. Although results vary according to the extent of dose reduction achieved, the majority of studies suggest that confident exclusion of calculi measuring $>4 \mathrm{~mm}$ in diameter is possible with most low-dose imaging protocols [14-24, 40, $41]$.

\section{Characterization of Urinary Calculi}

The attenuation value of different subtypes of renal calculi overlaps greatly on conventional single-energy CT datasets 


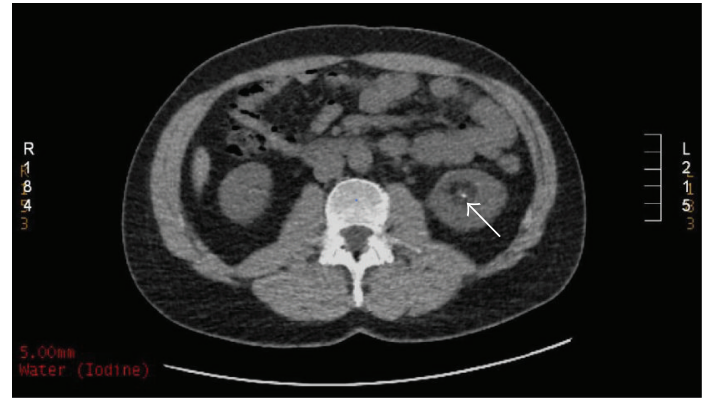

(a)

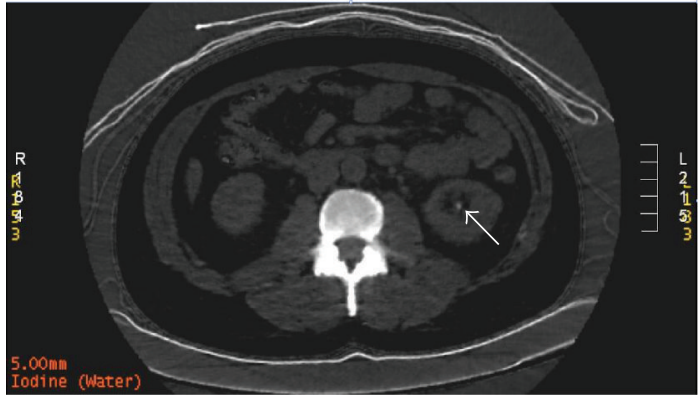

(b)

Figure 2: A 34-year-old male presenting with left flank pain. Axial dual energy noncontrast CT. (a) shows a 3 mm calculus in the left renal pelvis on iodine and (b) water-based attenuation. As the calculus is visible on both imaging techniques, this indicates high-molecular-weight elements. This calculus proved to be predominantly calcium based.

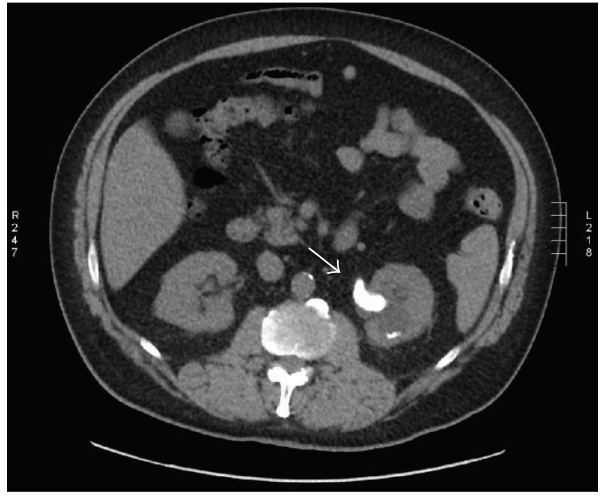

(a)

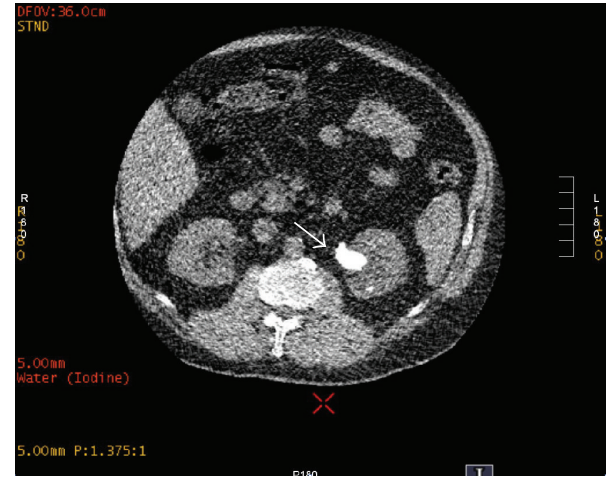

(c)

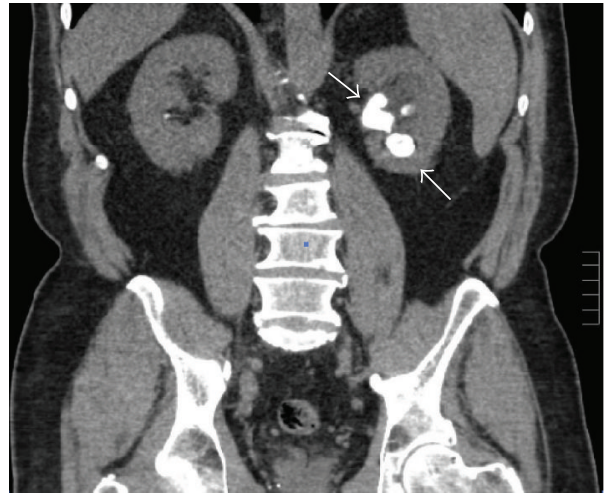

(b)

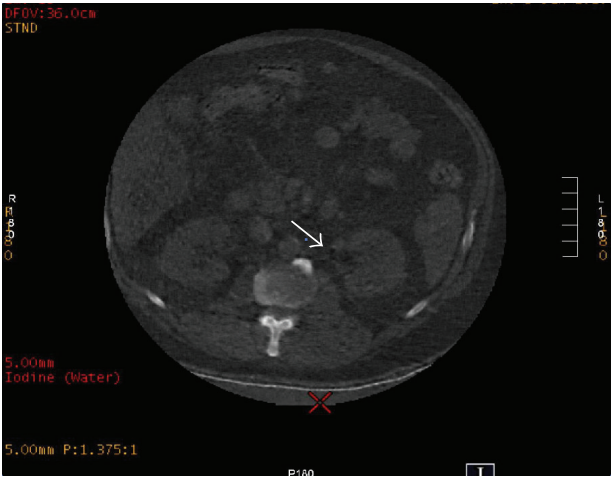

(d)

Figure 3: A 42-year-old male with a history of renal calculi presenting with left flank pain. Noncontrast CT for the assessment of renal calculi in axial (a) and coronal (b) reformats. These images clearly show a large calculus in the left renal pelvis extending into the upper ureter as well as a second large calculus in the lower pole of the left kidney (arrows). Axial dual energy noncontrast CT images in the same patient showing iodine- (c) and water- (d) based attenuation. The calculus is visible on the iodine-attenuated image (c) and is unchanged when compared to the standard CT. However, the water-attenuated image (d) shows "dropout," and the calculus is no longer visible. This indicates the presence of low-molecular-weight elements. Post removal, this stone proved to be predominantly composed of urate.

[42], but their attenuation values differ significantly when imaged with high- and low-energy CT. Uric acid stones, which are predominantly composed of low-molecularweight elements (oxygen, carbon, and nitrogen), have different X-ray attenuation properties at high- and low-energy CT compared with other types of renal calculi such as calcium oxalate, hydroxyapatite, or cysteine stones. These stones are composed of high-molecular-weight elements (phosphorus, calcium, and sulfur) and therefore, as a consequence, will have a higher Hounsfield unit value at lower-energy CT.

This characteristic difference in attenuation with dualenergy imaging (i.e., contemporaneous CT scanning at 80 
and $120-140 \mathrm{Kv}$ ) may potentially allow accurate determination of stone composition which may facilitate more appropriate management in patients with uric acid (UA) containing calculi who may benefit from medical management and those with cystine and certain calcium stones which may be more resistant to shock wave lithotripsy [43] (see Figures 2-3).

Studies involving dual-energy CT have proven the accuracy of this technique in both ex and in vivo studies [44-46].

Accuracy of dual-energy imaging appears however to also be dependent on stone size. In an in vivo study conducted by Manglaviti et al., where there was disagreement between the chemical composition analysis in urinary tract calculi determined by dual-energy CT and the actual composition determined by crystallography, the stone diameter in each case was less than $1 \mathrm{~cm}$; in each discrepant case, mixed uric acid and hydroxyapatite calculi were misclassified as cystine and hydroxyapatite on dual-energy CT [46].

It should also be acknowledged that at present, dualenergy imaging is associated with higher doses of ionizing radiation when compared with single-energy CT [47]. Work is currently underway to reduce radiation exposure associated with dual-energy imaging, however, and Thomas et al. report successful differentiation between calcific, uric acid, and cystine containing calculi with an effective dose comparable to intravenous pyelography in nonobese patients $(2.7 \mathrm{mSv})$ [45]. Targeted dual-energy scanning of calculi can be incorporated into a standard noncontrast CT scan in a dose-efficient way as follows: a single-high-energy low-dose scan of the entire urinary tract can be performed followed by targeted low-energy scanning of the areas where calculi are located. Use of imaging strategies such as these has been shown to decrease effective dose, but early results indicate a sustained increase in dose (59\%) when compared with lowdose single-energy CT alone [48].

Another approach is using CT to determine the relative fragility of calcium oxalate stones. Zarse et al. describe the use of micro-CT to delineate the internal structure of such calculi, as opposed to simple Hounsfield measurements, in the assessment of calculus suitability for shockwave lithotripsy. Their research showed that patients with calcium oxalate stones of homogenous composition were less likely to benefit from lithotripsy treatment than those with calculi displaying visible internal structure on micro-CT. The latter group of patients was found to have stones that were amenable to medical treatment as opposed to the lithotripsyresistant calculi with homogenous internal architecture. The authors go on to suggest that pretreatment $\mathrm{CT}$ can be used to assess calculus fragility and that it is stone morphology, rather than X-ray attenuation, that better correlates with overall fragility [49].

\section{Conclusion}

As a result of CT dose reduction measures that have been outlined in this paper, a cancer risk that was small to begin with is being systematically reduced [50]. Average radiation exposures associated with CT scanning of the urinary tract for urinary tract calculi are likely to reduce further and may eventually reach doses similar to those currently encountered in plain radiography with the help of iterative image reconstruction and other techniques. The statistical risks associated with performing a clinically indicated CT will therefore be reduced, but individual justification for performing CT will still be required.

Noncontrast CT remains the best imaging modality for the detection of urinary calculi and it has a high negative predictive value in excluding calculi, measuring greater than $4 \mathrm{~mm}$. Unfortunately, its spatial resolution, particularly when low-dose CT protocols are instituted, indicates that it is not suitable for completely excluding submillimeter calculi and small stone fragments in patients who are postendourological stone removal.

\section{References}

[1] R. C. Smith, A. T. Rosenfield, K. A. Choe et al., "Acute flank pain: comparison of non-contrast-enhanced CT and intravenous urography," Radiology, vol. 194, no. 3, pp. 789794, 1995.

[2] O. F. Miller, S. K. Rineer, S. R. Reichard et al., "Prospective comparison of unenhanced spiral computed tomography and intravenous urogram in the evaluation of acute flank pain," Urology, vol. 52, no. 6, pp. 982-987, 1998.

[3] J. M. H. Teichman, "Acute renal colic from ureteral calculus," New England Journal of Medicine, vol. 350, no. 7, pp. 684-693, 2004.

[4] J. W. Segura, G. M. Preminger, D. G. Assimos et al., "Ureteral stones clinical guidelines panel summary report on the management of ureteral calculi," The Journal of Urology, vol. 158, no. 5, pp. 1915-1921, 1997.

[5] D. M. Coll, M. J. Varanelli, and R. C. Smith, "Relationship of spontaneous passage of ureteral calculi to stone size and location as revealed by unenhanced helical CT," American Journal of Roentgenology, vol. 178, no. 1, pp. 101-103, 2002.

[6] R. Sierakowski, B. Finlayson, and R. R. Landes, "The frequency of urolithiasis in hospital discharge diagnoses in the United States," Investigative Urology, vol. 15, no. 6, pp. 438-441, 1978.

[7] G. C. Curhan, W. C. Willett, E. B. Rimm, and M. J. Stampfer, "Family history and risk of kidney stones," Journal of the American Society of Nephrology, vol. 8, no. 10, pp. 1568-1573, 1997.

[8] V. Romero, H. Akpinar, and D. G. Assimos, "Kidneystones: a global pictureof prevalence, incidence, and associated risk factors," Reviews in Urology, vol. 12, no. 2-3, pp. e86-e96, 2010.

[9] M. N. Ferrandino, A. Bagrodia, S. A. Pierre et al., "Radiation exposure in the acute and short-term management of urolithiasis at 2 academic centers," The Journal of Urology, vol. 181, no. 2, pp. 668-673, 2009.

[10] T. R. Koenig, D. Wolff, F. A. Mettler, and L. K. Wagner, "Skin injuries from fluoroscopically guided procedures: part I, characteristics of radiation injury," American Journal of Roentgenology, vol. 177, no. 1, pp. 3-11, 2001.

[11] E. Cardis, M. Vrijheid, M. Blettner et al., "The 15-country collaborative study of cancer risk among radiation workers in the nuclear industry: estimates of radiation-related cancer risks," Radiation Research, vol. 167, no. 4, pp. 396-416, 2007.

[12] F. V. Coakley, R. Gould, B. M. Yeh, and R. L. Arenson, "CT radiation dose: What can you do right now in your practice?" 
American Journal of Roentgenology, vol. 196, no. 3, pp. 619$625,2011$.

[13] A. Berrington de González, M. Mahesh, K. P. Kim et al., "Projected cancer risks from computed tomographic scans performed in the United States in 2007," Archives of Internal Medicine, vol. 169, no. 22, pp. 2071-2077, 2009.

[14] W. Liu, S. J. Esler, B. J. Kenny, R. H. Goh, A. J. Rainbow, and G. W. Stevenson, "Low-dose nonenhanced helical CT of renal colic: assessment of ureteric stone detection and measurement of effective dose equivalent," Radiology, vol. 215, no. 1, pp. 51$54,2000$.

[15] J. P. Heneghan, K. A. McGuire, R. A. Leder, D. M. DeLong, T. Yoshizumi, and R. C. Nelson, "Helical CT for nephrolithiasis and ureterolithiasis: comparison of conventional and reduced radiation-dose techniques," Radiology, vol. 229, no. 2, pp. 575580, 2003.

[16] T. H. Mulkens, S. Daineffe, R. de Wijngaert et al., "Urinary stone disease: comparison of standard-dose and low-dose with 4D MDCT tube current modulation," American Journal of Roentgenology, vol. 188, no. 2, pp. 553-562, 2007.

[17] S. Tartari, R. Rizzati, R. Righi, A. Deledda, S. Terrani, and G. Benea, "Low-dose unenhanced CT protocols according to individual body size for evaluating suspected renal colic: cumulative radiation exposures," Radiologia Medica, vol. 115, no. 1, pp. 105-114, 2010.

[18] T. Meagher, V. P. Sukumar, J. Collingwood et al., "Low dose computed tomography in suspected acute renal colic," Clinical Radiology, vol. 56, no. 11, pp. 873-876, 2001.

[19] M. Hamm, E. Knöpfle, S. Wartenberg, F. Wawroschek, D. Weckermann, and R. Harzmann, "Low dose unenhanced helical computerized tomography for the evaluation of acute flank pain," The Journal of Urology, vol. 167, no. 4, pp. 16871691, 2002.

[20] D. Tack, S. Sourtzis, I. Delpierre, V. de Maertelaer, and P. A. Gevenois, "Low-dose unenhanced multidetector CT of patients with suspected renal colic," American Journal of Roentgenology, vol. 180, no. 2, pp. 305-311, 2003.

[21] E. Knöpfle, M. Hamm, S. Wartenberg, and K. Bohndorf, "CT in ureterolithiasis with a radiation dose equal to intravenous urography: results in 209 Patients," RoFo, vol. 175, no. 12, pp. 1667-1672, 2003.

[22] D. S. Katz, N. Venkataramanan, S. Napel, and F. G. Sommer, "Can low-dose unenhanced multidetector CT be used for routine evaluation of suspected renal colic?" American Journal of Roentgenology, vol. 180, no. 2, pp. 313-315, 2003.

[23] C. Kluner, P. A. Hein, O. Gratta et al., "Does ultra-low-dose $\mathrm{CT}$ with a radiation dose equivalent to that of KUB suffice to detect renal and ureteral calculi?" Journal of Computer Assisted Tomography, vol. 30, no. 1, pp. 44-50, 2006.

[24] B. S. Kim, I. K. Hwang, Y. W. Choi et al., "Low-dose and standard-dose unenhanced helical computed tomography for the assessment of acute renal colic: prospective comparative study," Acta Radiologica, vol. 46, no. 7, pp. 756-763, 2005.

[25] M. K. Kalra, M. M. Maher, T. L. Toth, R. S. Kamath, E. F. Halpern, and S. Saini, "Comparison of z-axis automatic tube current modulation technique with fixed tube current CT scanning of abdomen and pelvis," Radiology, vol. 232, no. 2, pp. 347-353, 2004.

[26] A. C. Silva, H. J. Lawder, A. Hara, J. Kujak, and W. Pavlicek, "Innovations in CT dose reduction strategy: application of the adaptive statistical iterative reconstruction algorithm," American Journal of Roentgenology, vol. 194, no. 1, pp. 191199, 2010.
[27] A. K. Hara, R. G. Paden, A. C. Silva, J. L. Kujak, H. J. Lawder, and W. Pavlicek, "Iterative reconstruction technique for reducing body radiation dose at CT: feasibility study," American Journal of Roentgenology, vol. 193, no. 3, pp. 764$771,2009$.

[28] S. T. Schindera, L. Diedrichsen, H. C. Müller et al., "Iterative reconstruction algorithm for abdominal multidetector CT at different tube voltages: assessment of diagnostic accuracy, image quality, and radiation dose in a phantom study," Radiology, vol. 260, no. 2, pp. 454-462, 2011.

[29] A. C. T. Martinsen, H. K. Sæther, P. K. Hol, D. R. Olsen, and P. Skaane, "Iterative reconstruction reduces abdominal CT dose," European Journal of Radiology, vol. 81, no. 7, pp. 1483-1487, 2012.

[30] L. M. Mitsumori, W. P. Shuman, J. M. Busey, O. Kolokythas, and K. M. Koprowicz, "Adaptive statistical iterative reconstruction versus filtered back projection in the same patient: 64 channel liver CT image quality and patient radiation dose," European Radiology, vol. 22, no. 1, pp. 138-143, 2012.

[31] A. Gervaise, B. Osemont, S. Lecocq et al., "CT image quality improvement using adaptive iterative dose reduction with wide-volume acquisition on 320-detector CT," European Radiology, vol. 22, no. 2, pp. 295-301, 2012.

[32] Y. Sagara, A. K. Hara, W. Pavlicek, A. C. Silva, R. G. Paden, and Q. Wu, "Abdominal CT: comparison of low-dose CT with adaptive statistical iterative reconstruction and routine-dose CT with filtered back projection in 53 patients," American Journal of Roentgenology, vol. 195, no. 3, pp. 713-719, 2010.

[33] S. Singh, M. K. Kalra, J. Hsieh et al., "Abdominal CT: comparison of adaptive statistical iterative and filtered back projection reconstruction techniques," Radiology, vol. 257, no. 2, pp. 373-383, 2010.

[34] A. R. Kambadakone, P. Prakash, P. F. Hahn, and D. V. Sahani, "Low-dose CT examinations in Crohn's disease: impact on image quality, diagnostic performance, and radiation dose," American Journal of Roentgenology, vol. 195, no. 1, pp. 78-88, 2010.

[35] A. Winklehner, C. Karlo, G. Puippe et al., "Raw data-based iterative reconstruction in body CTA: evaluation of radiation dose saving potential," European Radiology, vol. 21, no. 12, pp. 2521-2526, 2011.

[36] G. A. Vorona, R. C. Ceschin, B. L. Clayton, T. Sutcavage, S. S. Tadros, and A. Panigrahy, "Reducing abdominal CT radiation dose with the adaptive statistical iterative reconstruction technique in children: a feasibility study," Pediatric Radiology, vol. 41, no. 9, pp. 1174-1182, 2011.

[37] J. A. Levine, J. Neitlich, M. Verga, N. Dalrymple, and R. C. Smith, "Ureteral calculi in patients with flank pain: correlation of plain radiography with unenhanced helical CT," Radiology, vol. 204, no. 1, pp. 27-31, 1997.

[38] K. A. B. Fowler, J. A. Locken, J. H. Duchesne, and M. R. Williamson, "US for detecting renal calculi with nonenhanced CT as a reference standard," Radiology, vol. 222, no. 1, pp. 109113, 2002.

[39] D. H. Jin, G. R. Lamberton, D. R. Broome et al., "Effect of reduced radiation CT protocols on the detection of renal calculi," Radiology, vol. 255, no. 1, pp. 100-107, 2010.

[40] M. W. Ciaschini, E. M. Remer, M. E. Baker, M. Lieber, and B. R. Herts, "Urinary calculi: radiation dose reduction of 50\% and $75 \%$ at CT-effect on sensitivity," Radiology, vol. 251, no. 1, pp. 105-111, 2009. 
[41] B. Karmazyn, D. P. Frush, K. E. Applegate, C. Maxfield, M. D. Cohen, and R. P. Jones, "CT with a computersimulated dose reduction technique for detection of pediatric nephroureterolithiasis: comparison of standard and reduced radiation doses," American Journal of Roentgenology, vol. 192, no. 1, pp. 143-149, 2009.

[42] M. F. Bellin, R. Renard-Penna, P. Conort et al., "Helical CT evaluation of the chemical composition of urinary tract calculi with a discriminant analysis of CT-attenuation values and density," European Radiology, vol. 14, no. 11, pp. 2134-2140, 2004.

[43] M. Eiber, K. Holzapfel, M. Frimberger et al., "Targeted dualenergy single-source CT for characterisation of urinary calculi: experimental and clinical experience," European Radiology, vol. 22, no. 1, pp. 251-258, 2012.

[44] C. Thomas, O. Patschan, D. Ketelsen et al., "Dual-energy CT for the characterization of urinary calculi: in vitro and in vivo evaluation of a low-dose scanning protocol," European Radiology, vol. 19, no. 6, pp. 1553-1559, 2009.

[45] C. Thomas, M. Heuschmid, D. Schilling et al., "Urinary calculi composed of uric acid, cystine, and mineral salts: differentiation with dual-energy CT at a radiation dose comparable to that of intravenous pyelography," Radiology, vol. 257, no. 2, pp. 402-409, 2010.

[46] G. Manglaviti, S. Tresoldi, C. S. Guerrer et al., "In vivo evaluation of the chemical composition of urinary stones using dual-energy CT," American Journal of Roentgenology, vol. 197, no. 1, pp. W76-W83, 2011.

[47] A. Graser, T. R. C. Johnson, M. Bader et al., "Dual energy CT characterization of urinary calculi: initial in vitro and clinical experience," Investigative Radiology, vol. 43, no. 2, pp. 112119, 2008.

[48] A. C. Silva, B. G. Morse, A. K. Hara, R. G. Paden, N. Hongo, and W. Pavlicek, "Dual-energy (spectral) CT: applications in abdominal imaging," Radiographics, vol. 31, no. 4, pp. 10311046, 2011.

[49] C. A. Zarse, A. H. Tariq, E. J. Molly et al., "CT visible internal stone structure, but not Hounsfield unit value, of calcium oxalate monohydrate (COM) calculi predicts lithotripsy fragility in vitro," Urological Research, vol. 35, no. 4, pp. 201206, 2007.

[50] E. S. Amis, "CT radiation dose: trending in the right direction,” Radiology, vol. 261, no. 1, pp. 5-8, 2011. 


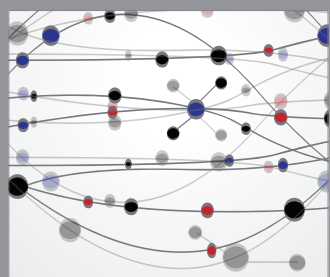

The Scientific World Journal
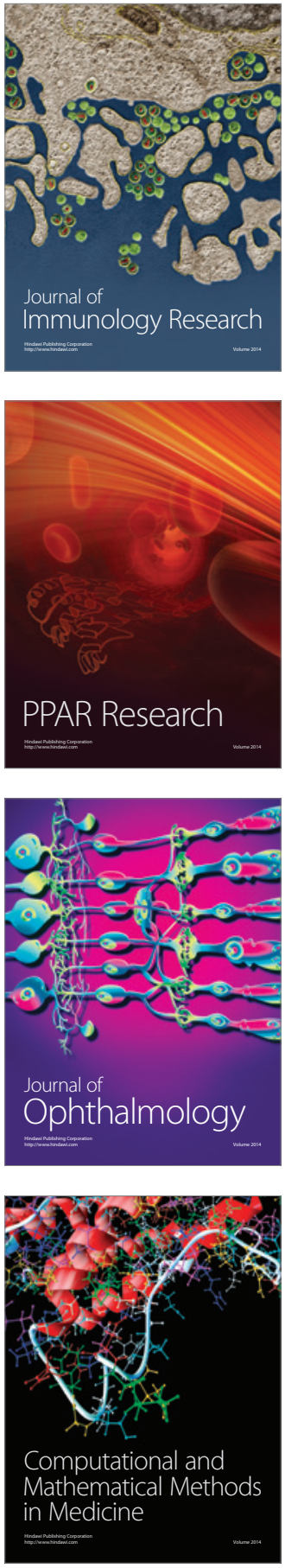

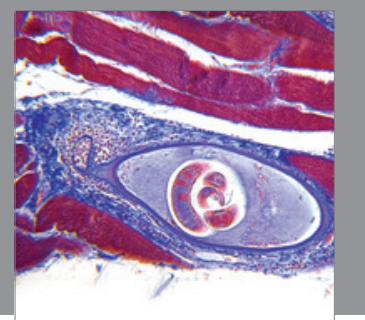

Gastroenterology

Research and Practice
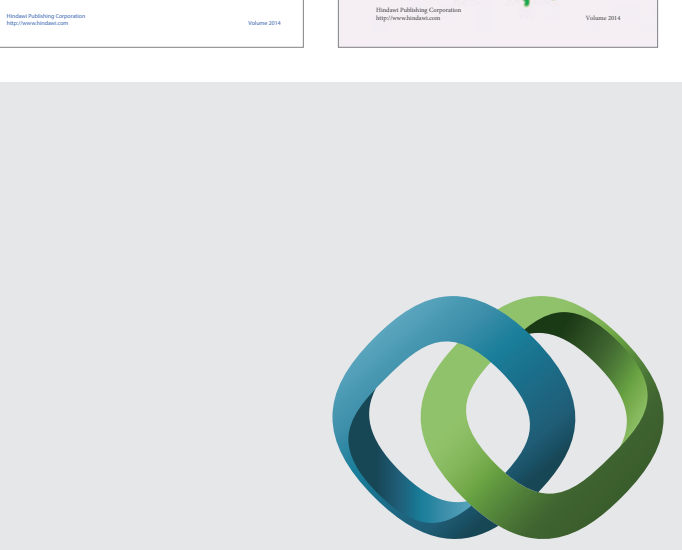

\section{Hindawi}

Submit your manuscripts at

http://www.hindawi.com
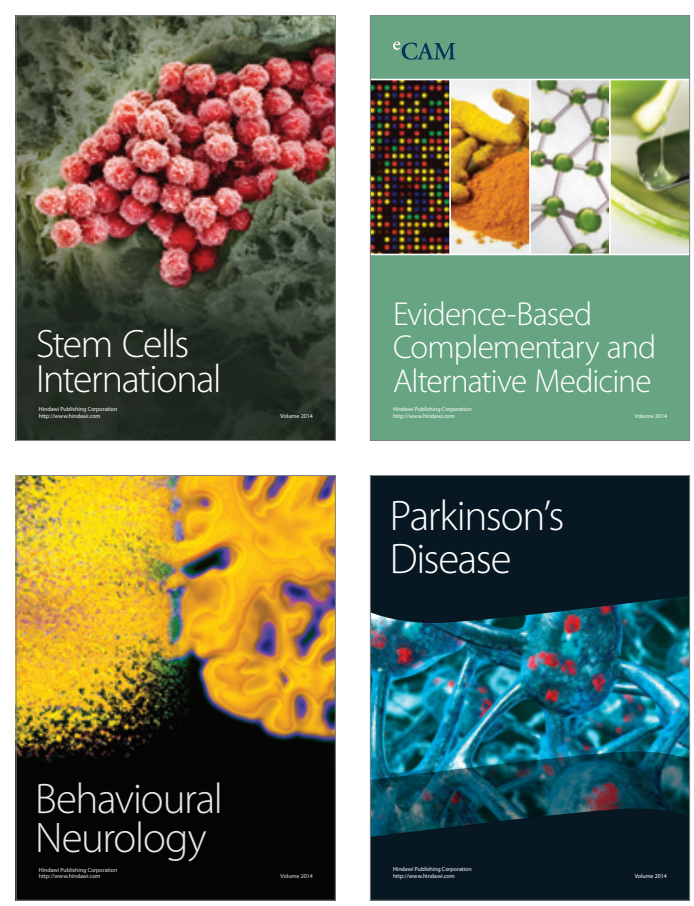

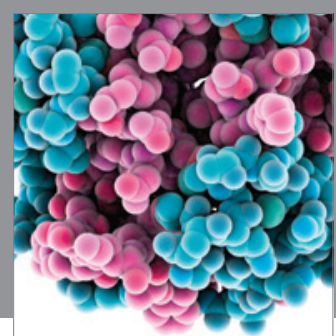

Journal of
Diabetes Research

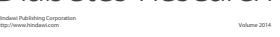

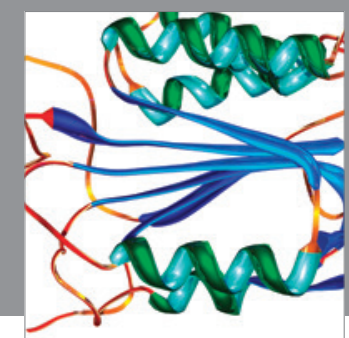

Disease Markers
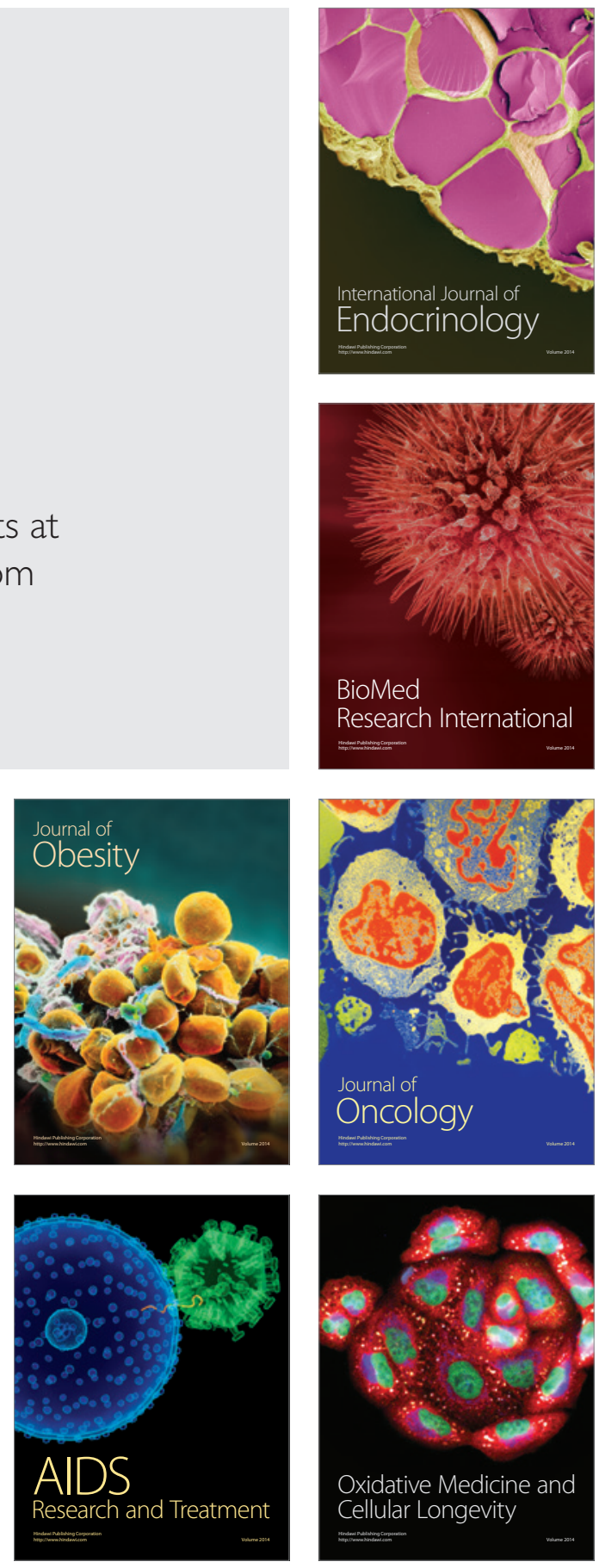